\title{
A Study on Relationship between Power of Adapter and Total Harmonic Distortion of Earphone's leaking Sound
}

\author{
Eun-Young Yi, Chan-Joong Jung and Myung-Jin Bae* \\ Department of Information and Telecommunication Engineering, Soongsil University \\ go6051@naver.com,cjjung99@hanmail.net,mjbae@ssu.ac.kr

\begin{abstract}
This paper researched relationship between Power of adapter and totat harmonic distortion of the open-type earphone. In the power of adapten even harnonic component is extremeley less and only the odd harmonics component is found. When analysed THD of leaking sound open-type earphone far from $1 \mathrm{~m}$, as aresult, even harmonic component was $2 \%$ and odd harmonic component was 35.5\%. THD of the diaphragm was affected by THD of electric signal.
\end{abstract}

Key words: THD, Even harmonic, Odd harmonic

\section{Introduction}

The advances in science and technologies have led way to the development of various equipment that made people's everyday lives more convenient. Although these advances allow our lives to become more affluent sometimes they may cause adverse effects to our health. One such example is noise. Noise is produced in various environments it is not limited to industrial settings it is present in our everyday lives, in our hobbies for example whether it be listening to musie or shooting. Interest in nois ${ }^{1} \mathrm{e}$ induced hearing loss or damage is increasing because the demographic of the sufferers of the condition have spread to a younger afe group from the traditionallaborers and the elderly that suffered from it [1].

The younger generation is often exposed to environments that are harmful to hearing due to leisurely activities such as going to dance clubs, going game room, attending concerts, band activity and ete. [2]. With the advance in technology there is an upsurge in the number of smart phone-and audio device, and there reports that the younger generations' usage of such devices caúse serious harm to their hearing.

The sound from portable electronic devices such as smart phone has a strong timber, with a wiđe maximum volume range of $78-136 \mathrm{~dB}$. Prolonged use of such devices may harm the sensitive of the ear in the $2000-4000 \mathrm{~Hz}$ range which is the most important range of the audio frequency needed to understand human speech [4].

These days students are using MP3, PMP, and smart-phones not only to listen to music but is using it watch videos, receive TV, and stream internet lectures, increasing their exposure to noise Listening to music at full volume through earphones with an MP3 results in 100-120dB if this volume sustained continuously, there is a high possibility of developing early symptom of noise induce hearing loss [5]. Therefore the leaked sounds from earphones should be recognized as noise and therefore needs to be systematically classified.

${ }^{*}$ Corresponding Author 
It has been reported that there is a correlation between the time spent using earphones and the stress levels of an individual, furthermore at $4000 \mathrm{~Hz}$ level the left war had a correlation between the preferred volume and hearing, whilst the right ear had a negative correlation between the stress level and the hearing [1]. Although there are many research about earphone users that are little to no research concerning sound leakage which affects the people around the earphone user. In order to investigate the sound leakage which affects the people around the earphone user in order to investigate the sound leakage from earphones, the conditions in which sound leakages occurs were identified. Earphones are used in both open elevator and etc. as shown in Table 2-1 below.

\section{Earphone Usage}

\subsection{Earphone Usage Location Survey}

Using earphone may cause sound leakage which may be perceived as nonse by people surrounding the user. However, there was no previous information concerning everyday use of earphones as well as information on the annoyance level of people being exposed to its noise. This paper surveys the everyday use of earphones as well as analyzes the reasons in which why the leaked sounds from earphones is heard and perceived as noise not only in the quiet environment but also in the bustling cityenvironment.

Table 2-1. Survey of Locations of Earphone Usage (Undergraduate Students 280 people) [1]

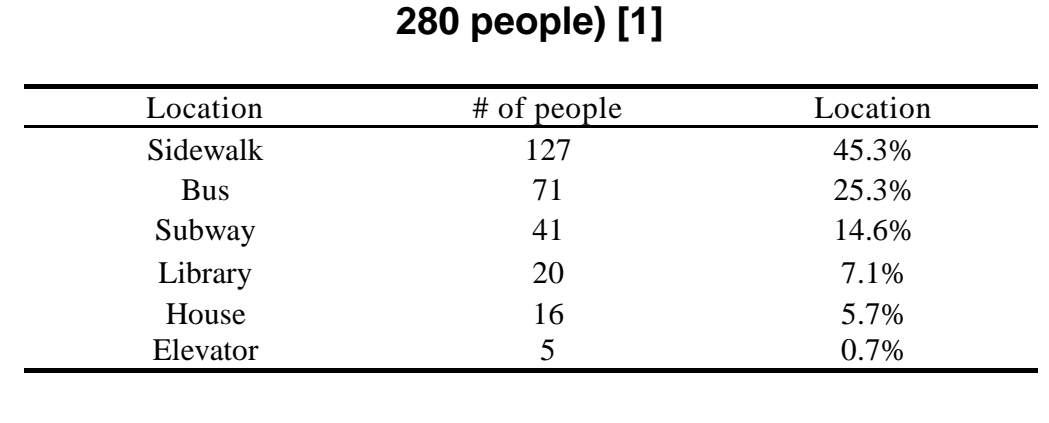

\subsection{Total Harmonic Distortion}

When a single frequency signal is applied to speaker, the total harmonic distortion (THD) is the percentile of sound pressure from the harmonics as opposed to the sound pressure from the base frequency. These distortions are caused by non-linearity in electro mechanical, vibration and acoustic systems. Distortions from each system might amplify or reduce each other's. As such in order to determine the THD of an earphone the relationship between each system must be analyzed exactly.

Distortions due to the non-linearity of the earphone are largely dependent on the deflection of the diaphragm. As the deflection of the diaphragm increase the distortion also has tendency to increase. In order to reduce the distortion rate, an enclosure is used. This experiment used a voice coil placed in the center of the circular diaphragm

$$
\begin{aligned}
& p_{n}: n^{\text {th }} \text { harmonic sound pressure response amplitude } \\
& p_{i}(f) \text { : Sound pressure at the receiving point derived from vibration system analysis. }
\end{aligned}
$$

Sound pressure response at frequency $\mathrm{f}$ is shown below. 
$p(t)=\sum_{n=1}^{\infty} p_{n} \cos \left[2 \pi f_{n} t+\phi_{n}+\beta_{i}\left(f_{n}\right)\right]$

$\beta_{i}(f)$ : Phase difference between the excitation force and sound pressure as derived by vibration system analysis.

$$
\begin{aligned}
& S P L=20 \log _{10} \frac{p_{s}}{20 \times 10^{-6}} \\
& T H D=\frac{\sqrt{a_{2}^{2}+a_{3}^{2}+a_{4}^{2}+a_{5}^{2}+a_{6}^{2}+\cdots \cdot}}{a_{1}} \times 100[\%]
\end{aligned}
$$

$n=2,3,4,5,6$ Sound pressure of harmonics.

\section{Figure 2-1 Voice Coil, Permanent Magnet, Edge Form}

The center has a vorce coil, the middle is the damper with the outermost acts as a permanent magnet The stiffnes of the damper determines the resonance requency. As the stiffness increse the resonanee frequency decrease, as the stiffness decrease the resonance frequency increase. Resomance is possible because the stiffness of the electrics acts as a inductance wherase comliance acts as a capacitance.

Table 2-2. Diaphram Pattern's Form and Shape

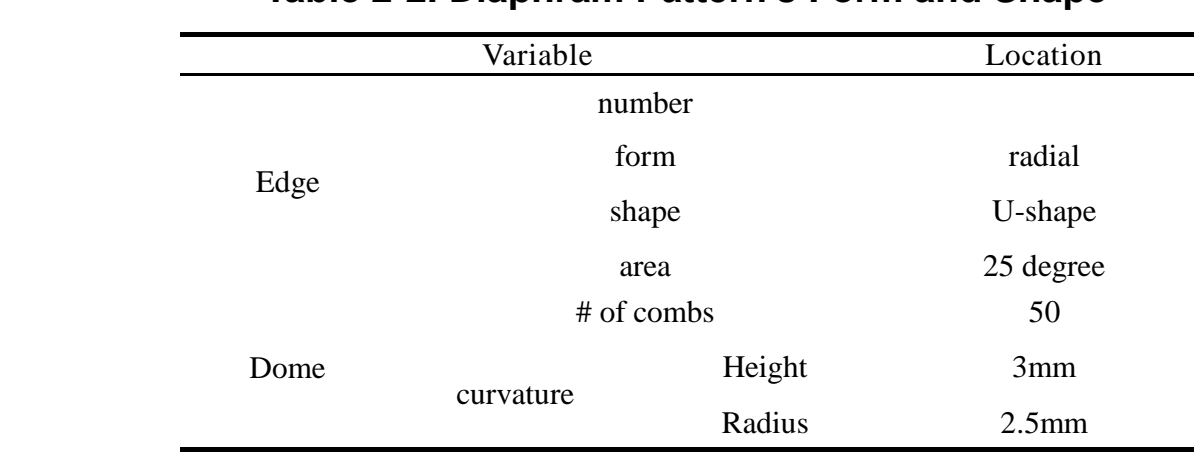

\subsection{Relationship Between Earphone and Total Harmonic Distortion}

Noise can be largely categorized into two categories, internal( in the earphone) and external (outside the earphone). There are two type of internal noise:harmonics due to the power source, and cross modulation due to the non-linearity of the semiconductor. This paper 
focused on the power source's effect on the harmonics. Research in THD began from electronic products.

These researches focused on estimating and reducing harmonic components that arose from an input of electric signal mathematically [6-9].

Table 2-3. Total Harmonic Distortion Using Adapter Input Power [unit: V]

\begin{tabular}{cccccc}
\hline \multicolumn{5}{c}{ Open type earphone(circular polymer diaphragm) } \\
\hline \multicolumn{5}{c}{ Odd harmonics } \\
harmonics & First & Third & Fifth & Second & Fourth \\
$500 \mathrm{~Hz}$ & 1 & 0.35 & 0.06 & 0.02 & 0.02 \\
$1000 \mathrm{~Hz}$ & 1 & 0.37 & 0.059 & 0.02
\end{tabular}

The total harmonic distrotion was $35.5 \%$ to a pure tone sine wave of $500 \mathrm{~Hz}$, and $37.5 \%$ at $1000 \mathrm{~Hz}$. Results showed that at $1000 \mathrm{~Hz}$ even harmonic components especially the $2^{\text {nd }}$ harmonic component was either not present or very small compared to the other harmonics, furthermore for the odd harmonics only the $3^{\text {t }}$ and harmonics, components were observed [10].

\section{Experiment and Result}

\subsection{Experiment}

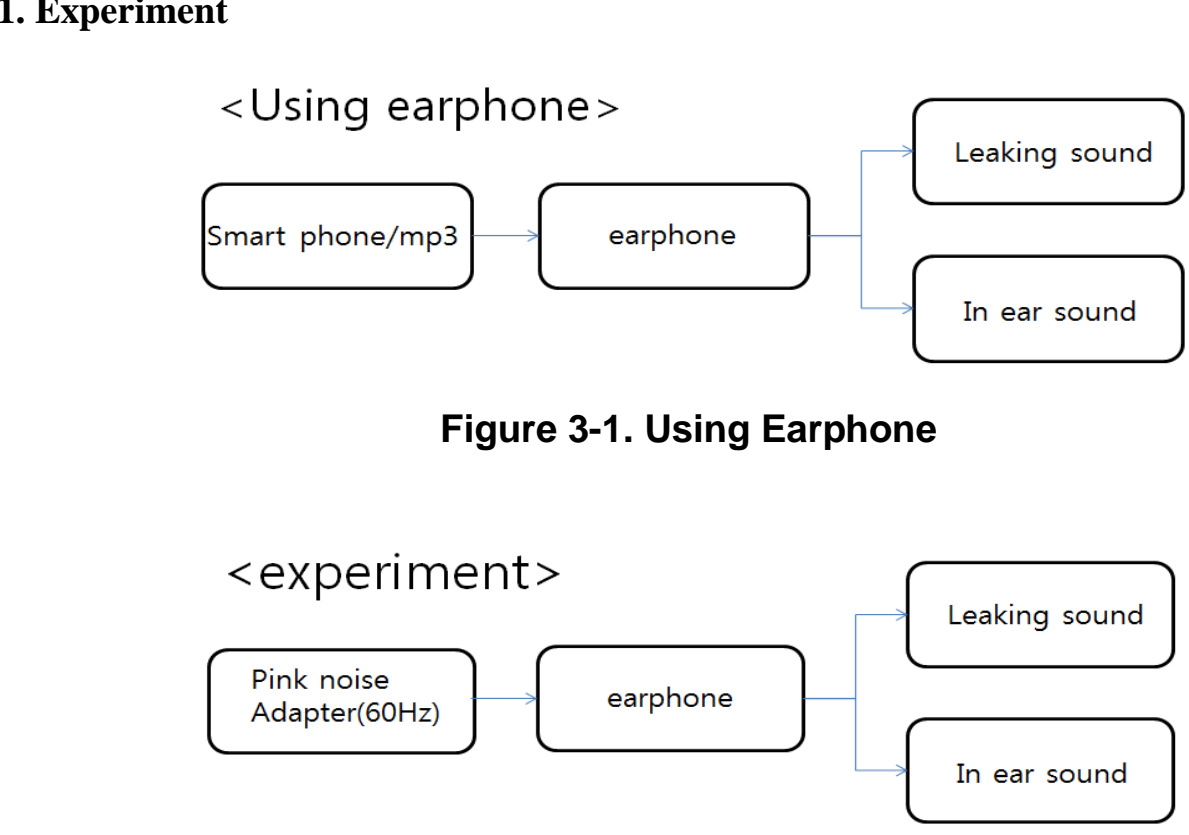

Figure 3-2. Experiment of Leaking Sound 


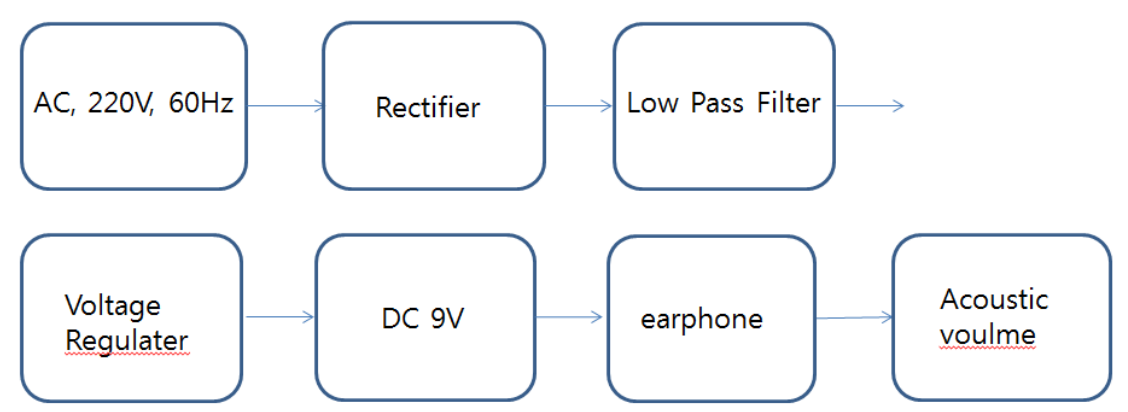

Figure 3-3. Structure of Adaptor

This paper compared between THD of adapter using common use power of $60 \mathrm{~Hz}$ and THD of earphone. The result that distortion of earphone depend on disfortion of adapter. Hereafter, various researches on power and maximum oscillation's action range of a diaphragm in earphone will be needed.

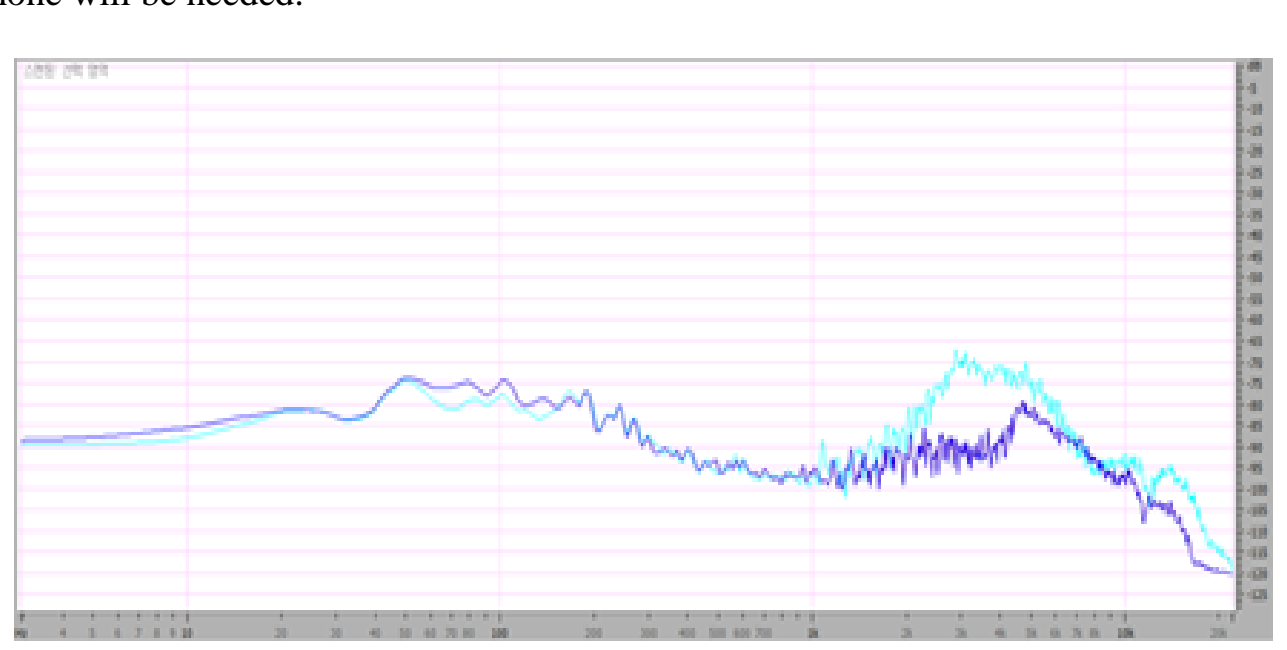

Figure 3-4. Leaking Sound of Pink Noise on Earphone

In this study, the leaking sound of two open-type earphone using a sound detector with the shape of an ear. As mentioned in the previous paper, the changes in frequency caused by the material type and thickness of the vibrating platform were observed in the $1^{\text {st }}$ resonance. The leaking sound in the frequency domain over $1000 \mathrm{~Hz}$ shows changes in the number of vent holes and their placement as illustrated in Figure.

Table 3-1. Leaking Sound from Open-type Earphone (Kang-Nam style)

\begin{tabular}{ccccc}
\hline \multicolumn{4}{c}{ "Kang Nam Style " leaking sound open from earphone } \\
\multicolumn{4}{c}{ 1m (dBA) Ambient noise : 33dBA } \\
\hline \multirow{4}{*}{ Medium Volume } & \multicolumn{2}{c}{ Maximum Volume } \\
Peak & Left & Right & Left & Right \\
Average & 42.1 & 43.7 & 47.3 & 48.2 \\
\hline
\end{tabular}


Leaking sound from open-type earphone(Kang-Nam style) is about average $37 \mathrm{dBA}$.

Table 3-2. Leaking Sound from Open-type Earphone (Shya Bang Shya Bang)

\begin{tabular}{|c|c|c|c|c|}
\hline \multicolumn{5}{|c|}{$\begin{array}{c}\text { "Shya Bang Shya Bang" leaking sound open from earphone } \\
\text { 1m (dBA) Ambient noise : 33dBA }\end{array}$} \\
\hline & \multicolumn{2}{|c|}{ Medium Volume } & \multicolumn{2}{|c|}{ Maximum Volume } \\
\hline & Left & Right & Left & Right \\
\hline Peak & 43.1 & 42.7 & 46.9 & 47.2 \\
\hline Average & 39 & 37.1 & 39.9 & 40.2 \\
\hline
\end{tabular}

\section{Conclusion}

Adapter used common use power supply mostly didn't affect distortion. Because theoretically, most of even harmonic component was eliminated Distortion of power supply can be caused by non-linearities of semiconductors. And the total harmonic distortion of earphone was generated due to get out of linear range of diaphragm's maximum oscillation range.

This paper compared between THD of adapter using common use power of $60 \mathrm{~Hz}$ and THD of earphone. The result that distortion of earphone depend on distortion of adapter. Hereafter, various researches on power and maximum oscillation's action range of a diaphragm in earphone will be needed.

\section{References}

[1] K. H and O. N. H.A. "Study on Relations among Use of Earphones, Stress, and Hearing Threshold in University Students" vol, 1, no. 12, issue 26, (2012), pp. 126-136.

[2] A Study on hearing threshold by noise exposure in university students using personal digital audio system. Unpublished master's thesis, Sun Moon University, Choong Chung Nam-Do.

[3] J. H. Chung, C.M. Des Roches, J. Meunier and R. D. Eavey, "Evaluation of noise induced hearing loss in young people using a web-based survey technique", Pediatrics, (2005), pp. 861-867.

[4] P. A. Hellstorm and Arelsson, "Sound level, hearing habits and hazards of using portable cassette players", Journal of Sound and Vibration, (1998), pp. 521-528.

[5] E. J. Kim, "Problems of Usage of Earphones, PCPs on Hearing in adolescents", Journal of Korean Society of School Health, (2009), pp. 107-118.

[6] V. Gosbell, S. Perera, and V. Smith "Harmonic distortion in the electric supply system", Integral Energy Power Qrality Centre, vol. 3, (2000) March.

[7] A. Opitiz C. Gherasim, M. Manana, C. J. Renedo, I. Eguiluz, and R. J. M. Belmans, "Total harmonic distortion decomposition depending on distortion origin", IEEE Transactions on Power delivery, vol. 1, no. 20 (2005), pp. 4.

[8] T. Komuro, S. Sobukawa, H. Sakayori, M. Kono and H. Kobayashi, "Total Harmonic distortion Measurement system of electric devices up to $100 \mathrm{MHz}$ with remarkable sensitivity”, IEEE Transactions.

\section{Authors}

\section{Yi-Eun Young}

Master Course in Engineering, Senior Researcher Assistant, Sori Sound Engineering Research Institute, Soongsil University. 


\section{Chan-Joong Jung}

Ph.D. in Engineering, Senior Researcher, Sori Sound Engineering Research Institute, Soongsil University.

\section{Myung-Jin Bae}

Ph.D. in Engineering, Professor, School of Electronics Engineering, Soongsil University.

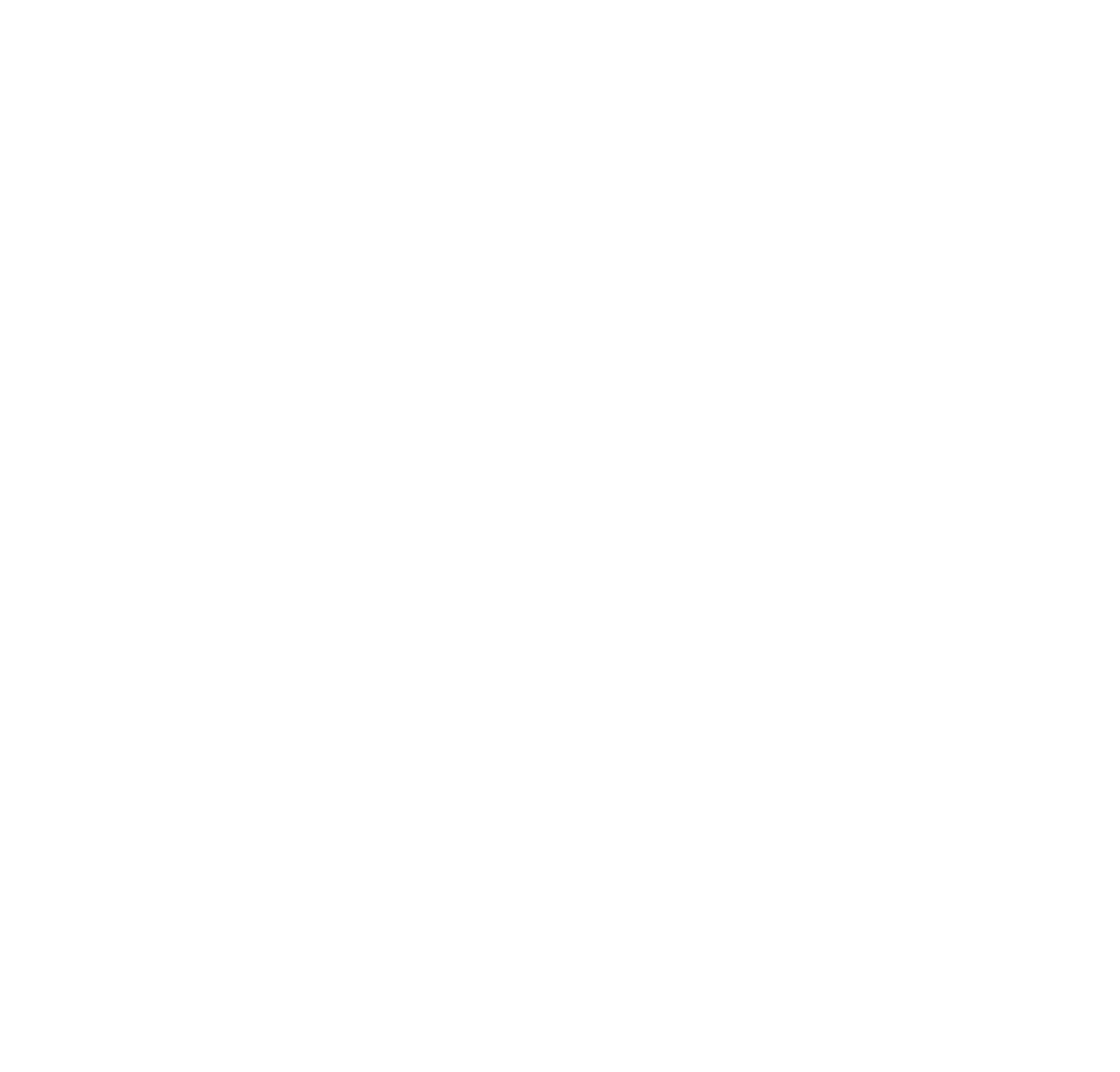


International Journal of Multimedia and Ubiquitous Engineering Vol.9, No.6 (2014)

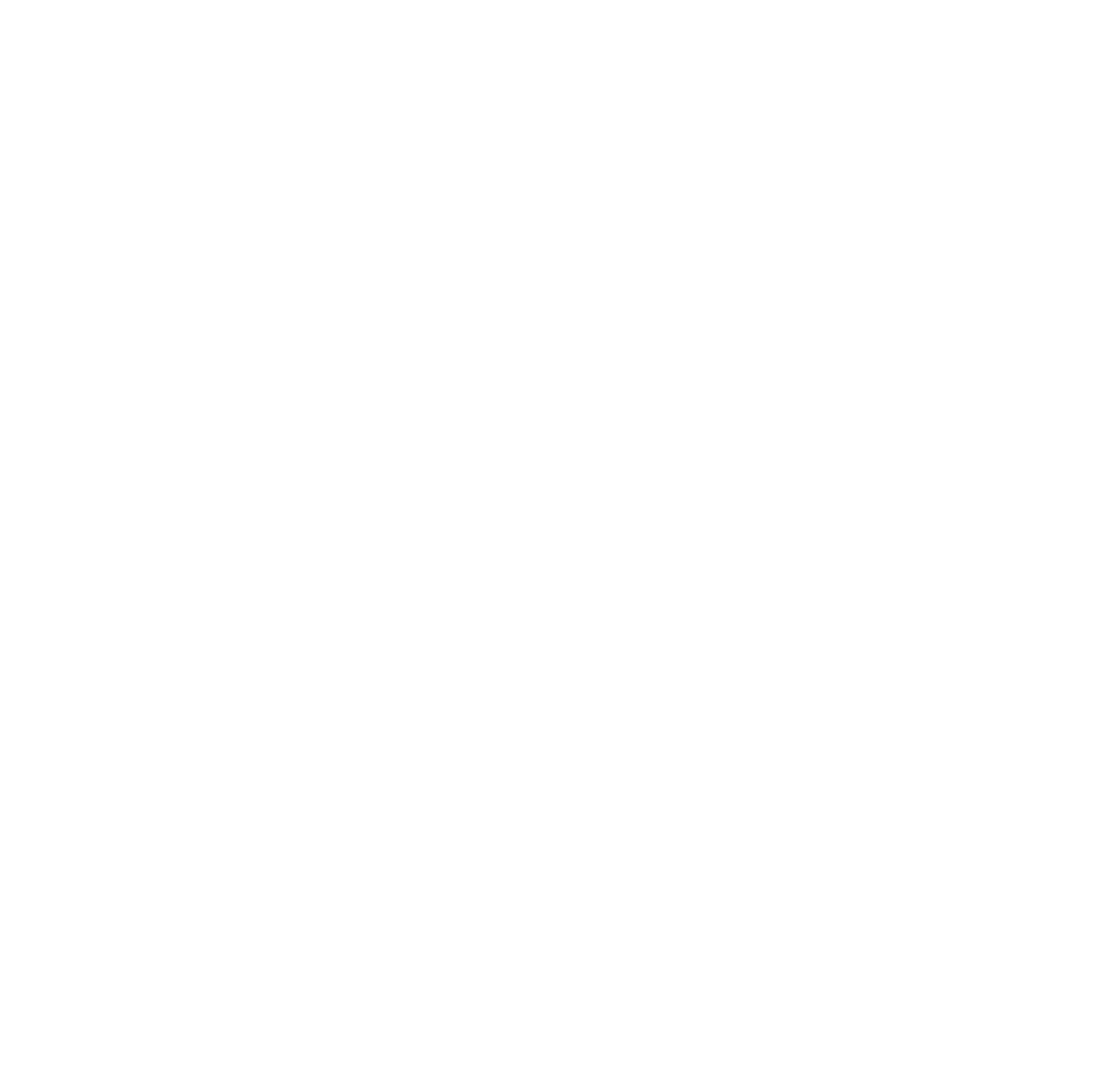

